\title{
Escala de Atitudes Frente ao Uso de Álcool: Descrevendo seus Parâmetros Psicométricos
}

Scale of Attitudes Toward Alcohol Use: Describing its Psychometric Parameters

Escala de Actitudes Frente al Uso de Alcohol:

Describiendo sus Parámetros Psicométricos

Valdiney V. Gouveia Universidade Federal da Paraíba

Carlos Eduardo Pimentel Universidade de Brasília

Paula Rachel Louro Leite \& Juliana Rodrigues de Albuquerque Universidade Federal da Paraíba

Thiago Araújo Bezerra da Costa Centro Universitário de João Pessoa 
Resumo: Este estudo objetivou conhecer evidências de validade e consistência interna da Escala de Atitudes frente ao Uso de Álcool (EAFUA). Participaram 259 estudantes universitários de João Pessoa, com idade média de 21 anos, a maioria mulheres $(65,6 \%)$, que responderam perguntas demográficas e preencheram a EAFUA. Esta é composta por quatro adjetivos bipolares (positivo/negativo; gosto/desgosto; bom/ruim e desejável/indesejável), respondidos em escala de 9 pontos $(-4$ a +4). Uma análise de componentes principais indicou um componente geral que explicou 82,3\% da variância total; seu alfa de Cronbach foi 0,93. A análise fatorial confirmatória reuniu índices de ajuste adequados para essa medida, corroborando sua estrutura unidimensional. Observaram-se diferenças de gênero nessa medida, que foi mais fortemente correlacionada com o uso de álcool (validade convergente) do que com o uso de maconha (validade discriminante). Esses resultados apóiam a adequação da EAFUA, portanto, esta poderá ser utilizada para conhecer o grau de favorabilidade ao uso de álcool entre adultos jovens.

Palavras-chave: Atitudes. Álcool. Gênero. Validade.

Abstract: This study aimed at knowing the evidences of validity and reliability of the attitudes toward the Alcohol Use Scale (ATAUS). The participants were 259 undergraduate students from João Pessoa, 21 years old average, most of them female (65.6\%). They answered to demographic questions and filled the ATAUS, composed by four bipolar adjectives (positive-negative, like-dislike, good-nasty, and desirable-undesirable) rating in a scale of 9-points $(-4$ to +4$)$. One component was observed according to the principal component analysis, accounting for $82.3 \%$ of the total variance, with Cronbach's alpha of 0.93 . Confirmatory factor analysis revealed adequate fit indexes, corroborating its one-factor structure. Moreover, it was observed the gender difference in the ATAUS' scores, and this measure was positively and more strongly correlated with the alcohol use (convergent validity) than with the marijuana use (discriminant validity). These findings support the psychometric adequacy of the ATAUS. Thus, it can be used in future studies to assess the degree of favorability toward alcohol among young adults.

Keywords: Attitudes. Alcohol. Gender. Validity.

Resumen: Este estudio tuvo como objetivo conocer evidencias de validez y consistencia interna de la Escala de Actitudes frente al Uso de Alcohol (EAFUA). Participaron 259 estudiantes universitarios de João Pessoa, con edad Media de 21 años, la mayoría mujeres (65,6\%), que contestaron a las preguntas demográficas y rellenaron la EAFUA. Ésta está compuesta por cuatro adjetivos bipolares (positivo/negativo; gusto/disgusto; bueno/malo y deseable/indeseable), contestados en escala de 9 puntos $(-4 \mathrm{a}+4)$. Un análisis de componentes principales indicó un componente general que explicó 82,3\% de la variancia total; su alfa de Cronbach fue 0,93. El análisis factorial confirmatorio reunió índices de ajuste adecuados para esa medida, corroborando su estructura unidimensional. Se observaron diferencias de género en esa medida, que fue más fuertemente correlacionada con el uso de alcohol (validez convergente) de lo que con el uso de marihuana (validez discriminante). Esos resultados apoyan la adecuación de la EAFUA, por tanto, ésta podrá ser utilizada para conocer el grado de favorabilidad al uso de alcohol entre adultos jóvenes.

Palabras clave: Aptitudes. Alcohol. Género. Validez.

A Organização Mundial de Saúde (OMS) calcula que aproximadamente $10-12 \%$ da população mundial e $11 \%$ da população brasileira sejam dependentes de bebidas alcoólicas; o álcool também é citado como responsável por cerca de $60 \%$ dos acidentes de trânsito e aparece em 70\% dos laudos referentes a mortes violentas (OMS, 1999). Estima-se que, no Ocidente, $90 \%$ dos adultos consumam bebidas alcoólicas (Ramos \& Woitowitz, 2004). O Brasil tem no consumo do álcool o responsável por mais de $10 \%$ de seus problemas totais de saúde (Meloni \& Laranjeira, 2004). De fato, os resultados do Centro Brasileiro de Informações sobre Drogas Psicotrópicas (CEBRID), frutos de levantamentos domiciliares realizados sobre o uso de drogas em mais de 100 dos maiores Municípios brasileiros (Carlini, Galduróz, Noto, \& Nappo, 2002; Carlini et al., 2007), retratam esse quadro. Percebeu-se um percentual de $12,3 \%$ de dependentes de álcool, o que corresponde a 7.939 brasileiros entre 12 e 65 anos de idade, sendo esse considerado o maior problema de saúde pública nacional; a situação na Região Nordeste é particularmente grave, com um quantitativo de dependentes próximo a $14 \%$, 
Apesar da tendência acentuada de pessoas em culturas ocidentais usarem álcool, essa prática pode ser diferente em razão do seu sexo. Em vários estudos no Brasil, por exemplo, foi observada maior predominância de alcoolismo entre os homens (Cardim, Assis, Sberze, Iguchi, \& Morgado, 1986; Carlini et al., 2002; Carlini et al., 2007; Galduróz \& Caetano, 2004). sendo que, em todas as regiões, observouse maior dependência das pessoas do sexo masculino (Carlini et al., 2007).

De acordo com Cocolo (2001), em outra pesquisa realizada pelo CEBRID em 450 instituições brasileiras de saúde, incluindo hospitais psiquiátricos, clínicas particulares e hospitais gerais, constatou-se que $90 \%$ das 726.429 internações por uso de drogas psicotrópicas no período de 1988 a 1999 decorreram do consumo de bebidas alcoólicas, sendo essa substância responsável por $94 \%$ das internações de pessoas maiores de 30 anos. Esse mesmo autor assevera que os dados do Ministério da Saúde indicam que a dependência de bebidas alcoólicas é a maior causa de internações psiquiátricas depois da esquizofrenia.

Outro fator de preocupação nacional diz respeito aos gastos exorbitantes produzidos com o consumo de álcool. Estimativas apontam um gasto anual de 7,3\% do Produto Interno Bruto (PIB) brasileiro com problemas advindos do uso de álcool no País, chegandose à quantia de 130 bilhões de reais por ano (Gallassi, Alvarenga, Andrade, \& Couttolenc, 2008). Conforme a Secretaria Nacional AntiDrogas (SENAD, 2008), entre 2002 e 2006, o Sistema Único de Saúde (SUS) gastou aproximadamente 37 milhões de reais com o tratamento de dependentes de bebidas alcoólicas e outras drogas. Adite-se a isso que, durante o mesmo período, mais $\mathrm{R} \$$ 4.317.251,59 foram gastos em procedimentos hospitalares de internações relacionadas com o uso de álcool e outras drogas. Na esfera da criminalidade, Duarte e Carlini-Cotrim (2000) analisaram 130 processos de homicídios ocorridos em Curitiba entre 1990 e 1995. Os resultados mostraram que $53,6 \%$ das vítimas e $58,9 \%$ dos autores dos crimes estavam sob efeito de bebidas alcoólicas no momento da ocorrência do crime. Além disso, o uso e o abuso do álcool envolvem diversos outros problemas sociais, além da violência e dos acidentes de trânsito (OMS, 1999), como, por exemplo, baixo desempenho escolar (Tavares, Béria, \& Lima, 2001), perda de emprego, vandalismo, problemas familiares, interpessoais e financeiros (Meloni \& Laranjeira, 2004) e abuso de menores (Habigzang, Koller, Azevedo, \& Machado, 2005).

Apesar da tendência acentuada de pessoas em culturas ocidentais usarem álcool, essa prática pode ser diferente em razão do seu sexo. Em vários estudos no Brasil, por exemplo, foi observada maior predominância de alcoolismo entre os homens (Cardim, Assis, Sberze, Iguchi, \& Morgado, 1986; Carlini et al., 2002; Carlini et al., 2007; Galduróz \& Caetano, 2004). No Chile e nos Estados Unidos, houve uma porcentagem similar entre homens e mulheres que usam álcool, porém, na Colômbia, o número de homens que fazem uso de bebidas alcoólicas é o dobro do de mulheres (Carlini et al., 2002). No Nordeste brasileiro, especificamente, a procura e a utilização de bebidas alcoólicas são geralmente mais freqüentes por parte dos homens (Carlini et al., 2002, 2007). Em João Pessoa, Gouveia, Vasconcelos e Jesus (1999) observaram que a variável sexo explica cerca de $18 \%$ da variabilidade de resposta ao CAGE, que avalia a possibilidade de a pessoa ser uma potencial bebedora-problema.

De acordo com o que antes se comentou, parece evidente a problemática do consumo de álcool e o predomínio, ao menos no contexto brasileiro, do uso por parte dos homens. Contudo, o que dizer acerca das atitudes frente ao álcool? Como a seguir será explicitado, as atitudes podem ter um papel preponderante nesse contexto, favorecendo a elaboração de programas de prevenção e/ou combate ao consumo excessivo do álcool. Portanto, parece pertinente dimensionar esse aspecto e conhecer também a forma de medi-lo. 


\section{Atitudes frente ao uso de álcool: evidências empíricas e medida}

Diversos modelos na Psicologia têm sido aplicados para explicar comportamentos no âmbito da saúde, como o uso de álcool e outras substâncias psicoativas (Dela Coleta, 2004; Hawkins, Catalano, \& Miller, 1992; Petraits, Flay, \& Miller, 1995). Pesquisas têm demonstrado que as atitudes são preditoras de comportamentos (Holland, Verplank, \& van Knippenberg, 2002), e atitudes frente ao uso de substâncias lícitas ou ilícitas predizem consistentemente o comportamento de uso (Ajzen, 2001; Hawkins et al., 1992; Holland et al., 2002; Petraits et al., 1995; Simons \& Gaher, 2004; Simons \& Carey, 1998, 2000). Nessa direção, isto é, considerando as atitudes, a teoria da ação racional explica que o uso de álcool é determinado por intenções e atitudes frente ao uso, além de normas sociais a respeito (Ajzen \& Fishbein, 1980; Fishbein \& Ajzen, 1975; Hays, 1985; Schlegel, D'Avernas, Zanna, DiTecco, \& Manske, 1987).

A importância das atitudes fica evidente também no estudo de Simons e Gaher (2004). Realizando um estudo prospectivo com estudantes universitários, esses autores verificaram que as atitudes positivas frente ao uso de álcool (Tempo 1) se correlacionaram com o seu consumo (Tempo 2). Essas atitudes também se correlacionaram com os problemas correlatos ao uso de álcool, e as atitudes frente ao não-uso moderaram a relação entre as atitudes frente ao uso e os problemas relativos ao uso. Nesse sentido, parece plausível pensar que, conhecendo tais atitudes, seria um passo importante para mudar comportamentos por meio de intervenções específicas, como a própria mudança de atitudes por comunicações persuasivas (Crano \& Prislin, 2006; Scott, 1996).
Frente ao que se comentou, parece evidente que contar com medidas de atitudes frente ao uso de álcool seja essencial. Procurando na literatura brasileira, foi possível encontrar ao menos dois instrumentos a respeito:

Escala de Atitudes frente ao Álcool, Alcoolismo e Alcoolista (Vargas, 2005): compreende 96 itens, respondidos em escala de cinco pontos, tipo Likert, variando de $\mathbf{1}=$ Discordo totalmente a $\mathbf{5}$ = Concordo totalmente. Esse instrumento abrange cinco fatores: I Trabalhar e relacionar-se com o alcoolista (por exemplo, o alcoolista é um irresponsável; o alcoolista é grosso, agressivo e mal-educado; Alfa $=0,92$ ), II - Etiologia (por exemplo, penso que fatores hereditários influenciam no uso do álcool; alcoolistas são pessoas que buscam na bebida soluções para problemas afetivos; Alfa = 0,87), III - Doença (por exemplo, o alcoolismo é responsável pela maioria dos acidentes; o alcoolista é um doente; Alfa $=0,74), \mathbf{I V}$ - Repercussões decorrentes do uso/abuso de álcool (por exemplo, a bebida alcoólica altera o estado emocional; a maioria dos alcoolistas acaba só; Alfa $=0,78$ ) e $\mathbf{V}$ - A bebida alcoólica (por exemplo, penso que as pessoas têm o direito de beber se elas quiserem; a bebida alcoólica é agradável e traz bem-estar; Alfa $=0,48$ ). Este último, provavelmente, talvez seja o fator mais claramente relacionado com atitudes frente ao consumo de álcool. Contudo, sua baixa consistência interna (Alfa de Cronbach) impõe restrições ao seu uso.

Questionário para Detectar Potencial Uso de Drogas entre Adolescentes (Problem Oriented Screening Instrument for Teenagers [POSIT]) (Coelho Júnior, Gontiès, \& Gouveia, 2003). Compreende a adaptação brasileira uma medida construída nos Estados Unidos e depois validada no México (Marino, González-Forteza, Andrade, \& MedinaMora, 1998). Originalmente, reunia 81 itens, respondidos em escala dicotômica ( $\operatorname{sim}$ ou não); na versão brasileira, ficou formada 
por 72 itens, adotando-se a mesma escala de resposta. Os itens foram distribuídos em sete fatores: I - Potencial consumo de álcool e maconha (Alfa $=0,80$ ), II Delinqüência juvenil compartilhada (Alfa = 0,71), III - Desequilíbrio emocional (Alfa $=0,72)$, IV - Conduta anti-social (Alfa $=$ $0,72), \mathbf{V}$ - Dificuldades no relacionamento com os pais/responsáveis (Alfa $=0,66$ ), VI - Dificuldades de aprendizagem (Alfa $=$ 0,66 ) e VII - Trabalho e desemprego (Alfa = 0,65 ). Claramente, apenas o primeiro fator se refere ao álcool, embora não exclusivamente (por exemplo, seus familiares ou amigos the disseram alguma vez que deve diminuir o uso de bebidas alcoólicas ou drogas? Começou a consumir maior quantidade de álcool ou drogas para obter o efeito que deseja?).

Percebe-se, pois, que as medidas encontradas são extensas, formadas por múltiplos itens, não se restringindo às atitudes frente ao álcool. Contrariamente, o instrumento de Vargas (2005) coloca maior ênfase no usuário contínuo do álcool, ou seja, no alcoolista. Desse modo, parece mais uma medida de preconceito frente a esse ator do que propriamente de atitudes frente às bebidas alcoólicas. Por outro lado, o instrumento empregado por Coelho Júnior et al. (2003) não se restringe ao álcool, fazendo referência nos mesmos itens à maconha e a outras drogas não denominadas. Além disso, o tipo de escala de resposta empregado nesse último caso é dicotômico, o que pode reduzir a variabilidade de resposta e mascarar a correlação do construto avaliado com respeito a outros.

Diante do contexto apresentado, pareceu escusável pensar em testar uma medida curta, focada unicamente em atitudes, procurando avaliar como as pessoas pensam, sentem ou reagem a estar sob uso de álcool. Esse aspecto constituiu o objetivo principal do presente artigo, que procurou reunir evidências de validade e consistência interna da medida elaborada. Esta partiu da concepção adotada para a Escala de Atitudes Frente ao Uso de Maconha (EAFUM), desenvolvida por Simons e Carey (2000) e adaptada para o contexto brasileiro por Gouveia, Pimentel, Queiroga, Meira e Jesus (2005). Em síntese, consiste em uma medida de diferencial semântico, contrastando quatro pares de adjetivos (positivo/negativo, agradável/desagradável, bom/ruim e desejável/indesejável) situados nos extremos de uma escala de respostas de 9 pontos. Essa medida pautou-se na conceituação de atitudes globais como "avaliações gerais", mensuradas em uma escala de intervalos (Crites, Fabrigar, \& Petty, 1994).

Consistentemente, comprovou-se a natureza unifatorial da EAFUM, que apresentou alfas de Cronbach superiores a 0,90 (Gouveia et al., 2005; Simons \& Carey, 2000). Recentemente, Gouveia, Pimentel, Medeiros, Gouveia e Palmeira (2007) procuraram conhecer a adequação de empregar esses pares de adjetivos para mensurar atitudes frente ao uso de drogas. A propósito, realizaram uma análise de componentes principais que indicou um único componente, explicando $79,3 \%$ da variância explicada total, com precisão, isto é, alfa de Cronbach de 0,91. Ademais, comprovou-se sua validade preditiva, evidenciando que as atitudes frente ao uso de drogas predisseram seu uso $(\beta=$ $0,17 ; O R=1,19, p=0,004)$.

Em resumo, o presente estudo pretendeu propor e reunir evidências sobre os parâmetros psicométricos da Escala de Atitudes Frente ao Uso de Álcool (EAFUA). Essa escala se propõe conhecer as atitudes frente ao álcool entre estudantes universitários, potencialmente implicados no uso e abuso de álcool. Levando-se em conta as pressões da vida universitária (Balan \& Campos, 2006; Domingos \& Domingos, 2005) e mesmo a faixa-etária em que se encontram os universitários, saindo da adolescência para 
a vida adulta, presume-se que esse grupo estudantil apresente risco eminente de uso e/ ou abuso de álcool, o que provavelmente tem motivado diversos estudos em que estes têm sido considerados (Balan \& Campos, 2006; Domingos \& Domingos, 2005; Kerr-Corrêa, Andrade, Bassit, \& Boccuto, 1999; Simons \& Gaher, 2004; Syre, Pesa, \& Cockley, 1999).

$\mathrm{O}$ instrumento ora tratado tem a vantagem de ser breve, formado por adjetivos em lugar de frases. Dessa forma, pode se constituir em uma ferramenta útil em estudos transculturais, de vez que sua tradução é mais simples do que seria se formada por frases longas. Com isso, a integração dos dados no âmbito das atitudes frente às substâncias ficaria facilitada. Em razão dessa vantagem, considera-se que seja justificado o presente estudo. Seu propósito é reunir evidências sobre suas validades fatorial, preditiva e convergente-discriminante, além de conhecer se homens e mulheres diferem nas pontuações dessa medida.

\section{Método}

\section{Participantes}

Participaram deste estudo 259 estudantes universitários, a maioria de uma universidade pública $(94,6 \%)$ em João Pessoa (PB). Os participantes estavam matriculados em 14 diferentes cursos (administração, ciências contábeis, ciências sociais, computação, Direito, educação física, Engenharia civil, Engenharia mecânica, farmácia, fisioterapia, música, nutrição, pedagogia e Psicologia), sendo a maioria de ciências contábeis (32\%) e Psicologia (24,7\%). A idade variou de 18 a 26 anos $(M=20,8 ; D P=1,86)$, sendo a maioria do sexo feminino $(65,6 \%)$, solteira $(93,4 \%)$ e de classe socioeconômica média $(59,9 \%)$ ou média-baixa (29\%). Desse total, 46,3\% admitiram ser usuários de álcool. Essa amostra foi de conveniência (nãoprobabilística), tendo participado as pessoas que, presentes em sala de aula, concordaram em colaborar com o estudo.

\section{Instrumento}

Escala de Atitudes Frente ao Uso de Álcool (EAFUA): compreende um instrumento no estilo daqueles desenvolvidos por Crites et al. (1994) para medir atitudes em relação a vários objetos, mas adaptando sua escala de resposta de sete para nove pontos a fim de medir atitudes frente ao uso de maconha (Simons \& Carey, 2000). Baseia-se em escalas de diferencial semântico, consistindo em saber a avaliação global do jovem acerca do efeito de álcool. Os quatro pares de adjetivos (positivo/ negativo, agradável/desagradável, bom/ ruim e desejável/indesejável) são situados nos extremos do diferencial semântico. As pontuações variam de +4 a -4 , tendo sido recodificadas como se segue: $+4=1,+3$ $=2,+2=3,+1=4,0=5,-1=6,-2$ $=7,-3=8$ e $-4=9$, portanto, $1,2,3$ e 4 representam atitudes favoráveis, sendo 50 ponto neutro, enquanto as pontuações 6, 7, 8 e 9 indicam atitudes desfavoráveis frente ao uso. Nesse sentido, a maior pontuação indica atitude contrária ao uso de álcool. No final desse questionário, foram incluídas questões referentes a dados sociodemográficos (idade, sexo, estado civil, grau de religiosidade, classe socioeconômica percebida, universidade onde estuda e curso que faz). Perguntouse, ainda, se a pessoa fazia uso de álcool e maconha.

\section{Escala de Atitudes Frente ao Uso de Álcool (EAFUA)}

Instruções. Por favor, indique sua opinião acerca de "estar sob o efeito de álcool". Pode ser uso leve, moderado ou pesado. Você não precisa ter usado bebidas alcoólicas para responder essas questões. Por favor, indique sua avaliação global de "estar sob o efeito de álcool". Marque um X no quadro que melhor representar sua resposta. 


\section{Considero "estar sob o efeito de álcool"...}

\begin{tabular}{|c|c|c|c|c|c|c|c|c|c|c|}
\hline & 4 & 3 & 2 & 1 & $\mathbf{0}$ & -1 & -2 & -3 & -4 & \\
\hline \multirow[t]{2}{*}{ Positivo } & $\square$ & $\square$ & $\square$ & $\square$ & $\square$ & $\square$ & $\square$ & $\square$ & $\square$ & Negativo \\
\hline & 4 & 3 & 2 & 1 & $\mathbf{0}$ & -1 & -2 & -3 & -4 & \\
\hline \multirow[t]{2}{*}{ Agradável } & $\square$ & $\square$ & $\square$ & $\square$ & $\square$ & $\square$ & $\square$ & $\square$ & $\square$ & Desagradável \\
\hline & 4 & 3 & 2 & 1 & $\mathbf{0}$ & -1 & -2 & -3 & -4 & \\
\hline \multirow[t]{2}{*}{ Bom } & $\square$ & $\square$ & $\square$ & $\square$ & $\square$ & $\square$ & $\square$ & $\square$ & $\square$ & Ruim \\
\hline & 4 & 3 & 2 & 1 & $\mathbf{0}$ & -1 & -2 & -3 & -4 & \\
\hline Desejável & $\square$ & $\square$ & $\square$ & $\square$ & $\square$ & $\square$ & $\square$ & $\square$ & $\square$ & Indesejável \\
\hline
\end{tabular}

\section{Procedimento}

Os aplicadores dos instrumentos foram previamente instruídos a não intervir nas respostas dos participantes, para minimizar a possibilidade de viés. Os questionários, auto-administráveis, foram aplicados de forma coletiva nas salas de aula, bastando aos participantes seguir as instruções neles descritas. Os aplicadores permaneceram em sala atentos a eventuais dúvidas. Assegurou-se aos participantes o sigilo de suas respostas, sendo dada a instrução de que não se identificassem. Um Termo de Consentimento Livre e Esclarecido foi anexado ao bloco de medidas, sendo rubricado pelo participante. O estudo, portanto, foi conduzido conforme os princípios éticos da Declaração de Helsinki e de suas emendas, com o consentimento dos participantes. O tempo médio para concluir sua participação foi de 10 minutos. Respondidos os instrumentos, estes eram rapidamente checados e, finalmente, expressavam-se os agradecimentos aos estudantes e aos professores presentes.

\section{Análises dos dados}

O SPSS 15 foi utilizado para tabular e realizar as análises descritivas (medidas de tendência central e dispersão, distribuição de freqüência) e calcular correlações de Pearson. Inicialmente, realizou-se uma Análise de Componentes Principais (ACP), procurando checar a viabilidade de se considerar os itens representantes de uma dimensão. Posteriormente, realizou-se uma Análise Fatorial Confirmatória (AFC) com o AMOS 7, considerando a matriz de covariância e adotando o estimador ML (máxima verossimilhança). Os seguintes indicadores de ajuste foram tidos em conta (Byrne, 2001; Tabachnick \& Fidell, 2001; Van de Vijver \& Leung, 1997):

1) $O \chi^{2}$ (qui-quadrado), que é um indicador de qualidade do ajuste. Nesse caso, quanto maior seu valor, pior o modelo. Por isso, pode-se chamar de índice de "maldade de ajuste", em oposição aos índices de "bondade de ajuste" (qualidade de ajuste), a exemplo do GFI e AGFI, que são tratados a continuação. O nível de significância correspondente precisa ser superior a 0,05 para indicar ajuste do modelo aos dados.

2) $\mathrm{O} \chi^{2} / g$.l. (razão do qui-quadrado pelos graus de liberdade) é um índice subjetivo da adequação do modelo. Um valor entre 2 e 3 é interpretado como a expressão de um ajuste satisfatório, admitindo-se até 5 como indicação de ajuste aceitável.

3) O Índice de Qualidade de Ajuste (GFI), o Índice de Qualidade de Ajuste Ponderado (AGFI) e o 
Índice de Comparação do Modelo (CFI) permitem avaliar se o modelo explica satisfatoriamente os dados. Valores de GFI, AGFI e CFI iguais ou superiores a 0,90 indicam que o modelo é adequado, ou seja, ajusta-se aos dados.

4) A Raiz do Erro Médio Quadrático de Aproximação (RMSEA) é um indicador de ajuste que leva em conta os residuais; valores menores ou próximos a 0,08 indicam um ajuste adequado do modelo (a diferença entre o modelo teórico e os dados é mínima); aceita-se um valor de até 0,10.

\section{Resultados}

\section{Evidências de validade fatorial}

Prévia à realização em si da Análise de Componentes Principais (ACP), visando a conhecer a pertinência desse tipo de análises, consideraram-se dois indicadores que apóiam sua pertinência: Teste de Esfericidade de Bartlett $\left[\chi^{2}(6)=852,03, p<0,001\right]$ e KMO $(0,84)$. No passo seguinte, decidiu-se conhecer o número de componentes a extrair. Embora tenham sido mais comumente empregados na literatura os critérios de Kaiser (valor próprio $\geq 1$ ) e Cattell (distribuição gráfica dos valores próprios) para tomar essa decisão, recentemente, tem sido considerada a análise paralela como um procedimento mais robusto, motivo pelo qual se sugere adotá-lo nesta oportunidade (Hayton, Allen, \& Scarpello, 2004). Esse tipo de análise calcula valores próprios a partir de bancos simulados, tendo como referência os números de participantes $(n=259)$ e variáveis $(i=4)$ do banco de dados empíricos. Realizaram-se, portanto, 1.000 simulações, calculando os valores próprios que deveriam ser contrastados com os empíricos; a existência de um componente é determinada em razão de o valor próprio observado ser superior ao simulado.

Os valores próprios observados foram: 3,28, 0,34, 0,25 e 0,13, enquanto os simulados (valores médios) foram: 1,14, 1,04, 0,96, 0,86. Fica evidente, portanto, a pertinência de extrair um único componente. Nesse sentido, o componente único foi denominado atitudes frente ao uso de álcool. Como é possível observar na Tabela 1, este permitiu explicar $82 \%$ da variância total. As saturações de seus itens variaram de 0,86 (positivo/negativo) a 0,95 (bom/ruim), com valor médio de 0,90. A consistência interna (alfa de Cronbach) desse componente foi 0,93.

Tabela 1. Estrutura fatorial e precisão da EAFUA .

\begin{tabular}{lcc}
\hline Item / Adjetivos & Saturação & Comunalidade \\
\hline Bom - Ruim & 0,95 & 0,90 \\
Agradável - Desagradável & 0,91 & 0,83 \\
Desejável - Indesejável & 0,90 & 0,81 \\
Positivo - Negativo & 0,86 & 0,74 \\
\hline Valor próprio & 3,28 & \\
\% Variância total & 82,0 & \\
Alfa de Cronbach & 0,93 &
\end{tabular}


Finalmente, em razão da natureza exploratória e circunstancial da ACP, realizou-se uma AFC. Os resultados são sumarizados na Figura 1, a seguir. Todas as saturações (Lambdas) foram estatisticamente diferentes de zero $\left(\lambda^{1} 0, z>1,96, p<0,001\right)$. Esse modelo unifatorial apresentou os seguintes indicadores de ajuste: $\chi^{2}(2)=4,43, p=0,11, \chi^{2} / \mathrm{gl}=2,21$, GFI $=0,99$, AGFI $=0,96, \mathrm{CFI}=0,98$ e RMSEA $=0,07$. Parecem evidentes, portanto, a estrutura fatorial e a consistência interna dessa medida, restando, entretanto, conhecer sua validade preditiva.

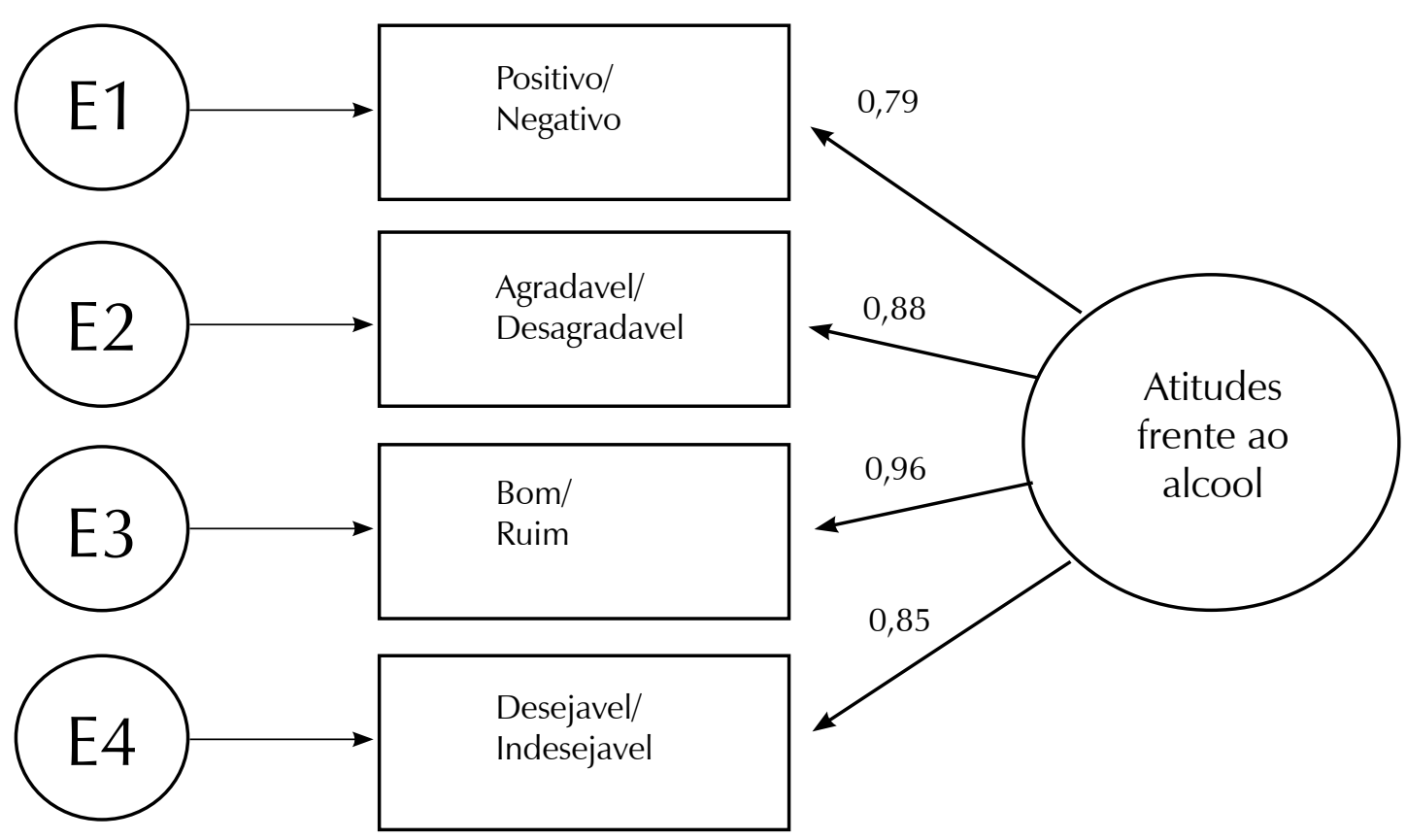

Figura 1. Confirmação da estrutura unifatorial da EAFUA.

\section{Validade preditiva e discriminante}

Como ficou evidenciado previamente, o consumo de álcool costuma ser mais freqüente entre os homens do que entre as mulheres. Isso pôde ser constatado no presente estudo, quando o quantitativo dos homens que indicaram consumir álcool (61,8\%) foi superior ao das mulheres $(38,2 \%), \chi^{2}(1)=13,11, p<0,001$. Portanto, se a EAFUA fosse adequada, conseguiria captar essa diferença também no plano das atitudes, o que foi constatado pelo fato de as mulheres apresentarem mais atitudes negativas frente ao álcool $(m=26,1, d p=8,95)$ do que os homens $(m=20,4, d p=9,54), t(257)=4,70, p<0,001$. Além disso, esperar-se-ia que essa medida apresentasse validade preditiva, com as pontuações dos participantes se correlacionando direta e significativamente com o fato de admitirem usar álcool. Isso foi observado $(r=0,68, p<$ 0,001). Por outro lado, embora se esperasse uma correlação significativa de tais pontuações com o consumo de maconha $(r=0,15, p<0,05)$, esse coeficiente deveria ser estatisticamente menor do que aquele observado para o consumo de álcool; isso foi corroborado quando foram comparados esses dois coeficientes $(z=7,66, p<0,001)$. Desse modo, indica-se a validade discriminante dessa medida. 


\section{Discussão}

O objetivo do presente estudo foi conhecer evidências de validade fatorial, validade convergente (preditiva)-discriminante e consistência interna da EAFUA. De acordo com as análises efetuadas, parece notória a adequação da proposta de Crites et al. (1994) para avaliar as atitudes frente a vários objetos sociais, como o uso de maconha (Gouveia et al., 2005) e drogas em geral (Gouveia et al., 2007). Isso também se aplica às atitudes frente ao álcool, como as que foram aqui medidas. Entretanto, devem-se ponderar as limitações potenciais deste estudo. Quanto à medida em si, é mais focada do que outras existentes na literatura (Coelho Júnior et al., 2003; Vargas, 2005), considerando um único tipo de droga (álcool). Nesse sentido, deve ser considerada unicamente quando o propósito for conhecer como as pessoas se posicionam frente às bebidas alcoólicas. Também deve ser ressaltado que a amostra considerada não permite generalizar os resultados acerca do consumo de drogas (álcool e maconha, especificamente), porém, lembrando, não foi esse o propósito do estudo, mas sim, conhecer os parâmetros métricos do instrumento em questão. Isso pareceu ter sido perfeitamente alcançado, sendo os resultados discutidos a seguir.

\section{Estrutura fatorial e consistência interna}

Os resultados para a EAFUA são similares àqueles encontrados quando da avaliação da EAFUM (Gouveia et al., 2005). Por exemplo, tanto por meio da Análise Fatorial Exploratória (ACP) como confirmatória (AFC, com estimador $\mathrm{ML}$ ), foi possível identificar o fator único das atitudes frente ao álcool. Os indicadores de ajuste se situaram no intervalo do que tem sido considerado satisfatório na literatura (Byrne, 2001; Van de Vijver \& Leung, 1997), e inclusive o c ${ }^{2}$, que costuma não funcionar com amostras acima de 200 participantes, apresentou valor não estatisticamente significativo, corroborando a estrutura unidimensional dessa medida. No tocante ao parâmetro precisão, especificamente consistência interna, o coeficiente observado para o componente/fator resultante é bastante satisfatório, estando bem acima do ponto de corte 0,70 proposto na literatura (Anastasi \& Urbina, 2000; Nunnally, 1991; Pasquali, 1999, 2003).

Uma das contribuições principais deste estudo, portanto, correspondeu à apresentação dos parâmetros da EAFUA. Essa medida de atitudes, definida como avaliações gerais expressas por meio de escalas tipo diferencial semântico (Crites et al., 1994; Simons \& Carey, 2000), corrobora resultados de estudos no contexto brasileiro que consideraram as atitudes frente à maconha (Gouveia et al., 2005) e às drogas (Gouveia et al., 2007) e também aqueles realizados em outras culturas (Crites et al., 1994; Simons \& Carey, 2000).

\section{Diferença de sexo e validade convergente-discriminante}

Quando se trata de consumo de bebidas alcoólicas, o sexo tem se revelado uma variável importante para explicar as taxas diferentes registradas, com predomínio do alcoolismo por parte dos homens (Cardim et al., 1986; Carlini et al., 2002, 2007; Galduróz \& Caetano, 2004). Esse quadro parece ainda mais evidente em cidades da Região Nordeste (Carlini et al., 2002; Gouveia et al., 1999), portanto, se a EAFUA fosse efetivamente adequada, deveria ser sensível a essa diferença de sexo. Isso foi claramente constatado, com as mulheres expressando mais atitudes negativas do que os homens.

Embora os consumos de álcool e maconha possam ser interdependentes (Coelho Júnior, 2001), essa não é uma correlação perfeita. Dessa forma, usuários de álcool podem ou não usar maconha, e vice-versa. Nesse contexto, 
em se tratando de uma medida de atitudes frente ao uso do álcool, seria esperado que as pontuações na EAFUA estivessem mais correlacionadas com o consumo dessa substância psicoativa (validade preditiva, convergente) do que com o uso de maconha (validade discriminante). Esse foi, portanto, um resultado teoricamente consistente que favoreceu um conhecimento adicional acerca da predição comportamental (uso de álcool) a partir das atitudes (Holland et al., 2002).

\section{Limitações do estudo}

Considera-se que este, como qualquer empreendimento científico, não é isento de aspectos que o limitam. No presente caso, uma classificação de tipos de usuários, como usuário recreativo, dependente de álcool ou alcoolista em grau severo, poderia contribuir para conhecer a capacidade preditiva da medida e avaliar também se sua estrutura fatorial seria invariante em razão dos grupos que fossem formados.

\section{Conclusão e direções futuras}

As atitudes compreendem um construto dos mais importantes da Psicologia social, uma ferramenta útil que pode ser empregada a partir de modelos teóricos para explicação do uso de álcool ou drogas (Dela Coleta, 2004, Hawkins et al., 1992; Petraits et al., 1995), como, por exemplo, a teoria da ação racional, a teoria da ação planejada e mesmo teorias mais integradas, que ofereçam explicações mais refinadas para o uso de bebidas alcoólicas (Hays, 1985). Medir tais atitudes é um passo preliminar, mas importante. Nesse sentido, oferecese aqui um instrumento para conhecer as atitudes das pessoas frente ao uso do álcool e mesmo para poder avaliar em que medida estas se modificam em razão de estratégias empregadas com esse fim (Crano \& Prislin, 2006; Scott, 1996). Empreendimentos dessa natureza podem ser eficazes para a diminuição dos problemas causados pelo uso e abuso do álcool neste país. Assim, confia-se oferecer nesta oportunidade uma contribuição aos estudos nessa área de interesse.

Em termos do que fazer no futuro, pesquisas adicionais poderão aportar evidências complementares acerca dos parâmetros da EAFUA. Poder-se-ia analisar, por exemplo, seus itens individualmente, por meio da teoria clássica dos testes ou da teoria de resposta ao item (Pasquali, 2003), com o fim de conhecer o poder discriminativo que estes apresentam. Outra estratégia será utilizar essa escala com as medidas de atitudes frente às drogas e à maconha; no caso, configurarse-á um questionário amplo ou uma bateria de escalas com o fim de avaliar atitudes e comportamentos frente a substâncias lícitas (uso de tabaco, por exemplo) e ilícitas (como a cocaína, o ecstasy ou o ácido lisérgico). Correlacioná-la com outras medidas com as quais, em princípio, deveria estar associada, será uma estratégia importante; no caso, listaram-se previamente duas dessas medidas (Coelho Júnior et al., 2003; Vargas, 2005). Conhecer a estabilidade temporal da EAFUA, isto é, sua precisão teste-reteste, também contribuirá para conhecer o quanto essa medida é estável, refletindo com precisão a pontuação dos participantes em diferentes momentos (Anastasi \& Urbina, 2000).

Não se descarta, ademais, a pertinência de contar com amostras de todo o território nacional, contudo, a consecução desse empreendimento deve motivar um programa mais amplo de pesquisa, visando, por exemplo, a entender melhor as atitudes e os comportamentos frente ao álcool assim como aos seus correlatos ou antecedentes, como os valores humanos e os traços de personalidade em diferentes grupos, como usuários e não usuários. Nesse cenário, em que se estima a inclusão de diversas medidas, a EAFUA tem a vantagem de ser curta, o que favorece sua 
aplicação em menor tempo e em diversos contextos.

Finalmente, além da mensuração de atitudes frente ao uso de álcool, novos estudos poderão focalizar as atitudes frente à experiência sem drogas, visto que estas, presumivelmente, agem como fator de proteção (assim como as atitudes negativas frente ao álcool), moderando a relação entre atitudes frente ao uso de álcool e problemas relacionados ao uso de álcool (Simons \& Gaher, 2004). Diversos estudos têm focalizado fatores de risco para o uso de drogas, como as atitudes favoráveis a essas substâncias (Hawkins et al., 1992;
Petraitis et al., 1995), sendo menos freqüentes aqueles que lidam ou que têm em conta os fatores de proteção. A propósito, Santos (2008) apresenta um vasto apanhado sobre fatores de proteção, pautado na hipótese do compromisso convencional e na afiliação social. No caso, destaca como fatores de proteção de comportamentos socialmente desviantes os valores humanos normativos (por exemplo, tradição, obediência), a identificação com grupos convencionais (por exemplo, pais, professores) e a afiliação religiosa (por exemplo, crenças religiosas, práticas religiosas).

Valdiney V. Gouveia*

Doutor em Psicologia Social. Professor da Universidade Federal da Paraíba, João Pessoa, PB - Brasil.

\section{Carlos Eduardo Pimentel}

Doutorando em Psicologia Social, do Trabalho e das Organizações, Universidade de Brasília,

Brasília, DF - Brasil.

E-mail: carlospimentel@unb.br

\section{Paula Rachel Louro Leite}

Psicóloga pela Universidade Federal da Paraíba, João Pessoa, PB - Brasil.

E-mail: paula_rachel_@hotmail.com

\section{Juliana Rodrigues de Albuquerque}

Estudante de psicologia na Universidade Federal da Paraíba, João Pessoa, PB - Brasil.

E-mail: july_albuquerque@hotmail.com

\section{Thiago Araújo Bezerra da Costa}

Psicólogo pelo Centro Universitário de João Pessoa, João Pessoa, PB - Brasil.

\section{*Endereço para envio de correspondência:}

Universidade Federal da Paraíba

CCHLA - Departamento de Psicologia - João Pessoa, PB - Brasil - CEP: 58051-900

E-mail:vvgouveia@gmail.com 


\section{Referências}

Ajzen, I. (2001). Nature and operation of attitudes. Annual Review of Psychology, 52(1), 27-58.

Ajzen, I., \& Fishbein, M. (1980). Understanding attitudes and predicting social behavior. Englewood Cliffs, NJ: Prentice-Hall.

Anastasi, A., \& Urbina, S. (2000). Testagem psicológica. Porto Alegre: Artes Médicas.

Balan, T. G., \& Campos, C. J. G. (2006). Padrão de consumo de bebidas alcoólicas entre graduandas de enfermagem de uma universidade estadual paulista. SMAD: Revista Eletrônica Saúde Mental Álcool Drogas, 2(2), 1-12. Disponível em: http://pepsic.bvs-psi.org.br/pdf/smad/v2n2/v2n2a03.pdf

Byrne, B. M. (2001). Structural equation modeling with Amos: Basic concepts, applications, and programming. London: Lawrence Erlbaum Associates.

Cardim, M. S., Assis, S. G., Sberze, M., Iguchi, T., \& Morgado, A. F. (1986). Epidemiologia descritiva do alcoolismo em grupos populacionais do Brasil. Cadernos de Saúde Pública, 2(2), 191-211.

Carlini, E. A., Galduróz, J. C. F., Noto, A. R., \& Nappo, S. A. (2002). I Levantamento domiciliar sobre o uso de drogas psicotrópicas no Brasil: estudo envolvendo as 107 maiores cidades do País - 2001. São Paulo: Centro Brasileiro de Informações sobre Drogas Psicotrópicas/Universidade Federal de São Paulo.

Carlini, E. A., Galduróz, J. C., Noto, A. R., Carlini, C. M., Oliveira, L. G., Nappo, S. A., Moura, Y. G., \& Sanchez, Z. V. D. M. (2007). Il levantamento domiciliar sobre o uso de drogas psicotrópicas no Brasil: estudo envolvendo as 108 maiores cidades do País - 2005. São Paulo: Páginas \& Letras.

Cocolo, A. (2001, setembro). Álcool é principal causa de internação por uso de drogas psicotrópicas. Jornal da Paulista, ano 14, 159. Recuperado em 28 de janeiro de 2008 de, http://www. unifesp.br/comunicacao/jpta/ed159/pesq6.htm

Coelho Júnior, L. L. (2001). Uso potencial de drogas em estudantes do ensino médio: suas correlações com as prioridades axiológicas. Dissertação de Mestrado. Departamento de Psicologia, Universidade Federal da Paraíba, João Pessoa.

Coelho Júnior, L. L., Gontiès, B., \& Gouveia, V. V. (2003). Questionário para detectar potencial uso de drogas entre adolescentes (Posit): adaptação brasileira. Jornal Brasileiro de Psiquiatria, 52, 109-116.

Crano,W. D., \& Prislin, R. (2006). Attitudes and persuasion. Annual Review of Psychology, 57, 345-374.

Crites, S. L., Fabrigar, L. R., \& Petty, R.E. (1994). Measuring the affective and cognitive properties of attitudes: Conceptual and methodological issues. Personality and Social Psychology Bulletin, 20(6), 619-634.

Dela Coleta, M. F. (Org.). (2004). Modelos para pesquisa e modificação de comportamentos de saúde. Taubaté, SP: Cabral.

Domingos, N. A. M. I., \& Domingos, J. C. V. (2005). Levantamento sobre o uso de álcool e drogas entre universitários. Revista Brasileira de Terapias Cognitivas, 1(1), 75-82. 


\section{Referências}

Duarte, P. C. A. V., \& Calini-Cotrim, B. (2000). Álcool e violência: estudo dos processos de homicídios julgados nos Tribunais de Júri de Curitiba, PR, entre 1995 e 1998. Jornal Brasileiro de Dependências Químicas, 1(1),17-25.

Fishbein, M., \& Ajzen, I. (1975). Belief, attitude, intention, and behavior: An introduction to theory and research. Reading, MA: Addison-Wesley.

Galassi, A. D., Alvarenga, P. G., Andrade, A. G., \& Couttollenc, B.F. (2008). Custos dos problemas causados pelo abuso do álcool. Revista de Psiquiatria Clínica, 35(Supl. 1), 25-30.

Galduróz, J. C. F., \& Caetano, R. (2004). Epidemiologia do uso do álcool no Brasil. Revista Brasileira de Psiquiatria, 26(Supl. 1), 3-6.

Gouveia, V. V., Pimentel, C. E., Medeiros, E. D., Gouveia, R. S V., \& Palmeira, J. (2007). Escala de atitudes frente ao uso de drogas: evidências de validade fatorial e preditiva. Jornal Brasileiro de Psiquiatria, 56(1), 53-59.

Gouveia, V. V., Pimentel, C. E., Queiroga, F., Meira, M., \& Jesus, G. R. (2005). Escala de atitudes frente ao uso de maconha: comprovação da sua validade de construto. Jornal Brasileiro de Psiquiatria, 54(1), 5-12.

Gouveia, V. V., Vasconcelos, T. C., \& Jesus, G. R. (1999). Índice de bebedores-problemas em João Pessoa: uma estimativa com base no CAGE. Temas, 56-57, 44-53.

Habigzang, L. F., Koller, S. H., Azevedo, G. A., \& Machado, P. X (2005). Abuso sexual infantil e dinâmica familiar: aspectos observados em processos jurídicos. Psicologia: Teoria e Pesquisa, 21(3), 341-348.

Hawkins, J. D., Catalano, R. F., \& Miller, J. Y. (1992). Risk and protective factors for alcohol and other drug problems in adolescence and early adulthood: Implications for substance abuse prevention. Psychological Bulletin, 112(1), 64-105.

Hays, R. (1985). An integrated value-expectancy theory of alcohol and other drug use. British Journal of Addiction, 80, 379-384.

Hayton, J. C., Allen, D. G., \& Scarpello, V. (2004). Factor retention decisions in exploratory factor analysis: A tutorial on parallel analysis. Organizational Research Methods, 7(2), 191-205.

Holland, R. W., Verplank, B., \& van Knippenberg, A. (2002). On the nature of attitude-behavior relations: The strong guide, the weak follow. European Journal of Social Psychology, 32, 869-872.

Kerr-Corrêa, F., Andrade, A. G., Bassit, A. Z., \& Boccuto, N. M V. F. (1999). Uso de álcool e drogas por estudantes de medicina da Unesp. Revista Brasileira de Psiquiatra, 21, 95-100.

Marino, M. C., González-Fortaleza, C., Andrade, P. E., \& MedinaMora, M. E. (1998).Validación de un cuestionario para detectar adolescentes con problemas por el uso de drogas. Salud Mental, 21, 21-36.

Meloni, J. N., \& Laranjeira, R. (2004). Custo social e de saúde do consumo do álcool. Revista Brasileira de Psiquiatria, 26(Supl. I), 7-10.

Nunnally, J. C. (1991). Teoría psicométrica. México, DF: Trillas.
Organização Mundial da Saúde. (1999). Global status report on alcohol. OMS: Genebra. Recuperado em 03 de novembro de 2008, dehttp://www.who.int/substance_abuse/publications/ en/GlobalAlcohol_overview.pdf

Pasquali, L. (1999). Instrumentos psicológicos: manual prático de elaboração. Brasília, DF: LabPAM/IBAPP.

Pasquali, L. (2003). Psicometria: teoria dos testes na psicologia e educação. Petrópolis, RJ: Vozes.

Petraits, J., Flay, B. R., \& Miller, T.Q. (1995). Reviewing theories of adolescent substance use: Organizing pieces in the puzzle. Psychological Bulletin, 117(1) 67-86.

Ramos, S. P., \& Woitowitz, A. B. (2004). Da cervejinha com os amigos à dependência do álcool: uma síntese sobre o que sabemos sobre esse percurso. Revista Brasileira de Psiquiatria, 26(Supl. I), 18-22.

Santos, W. S. (2008). Explicando comportamentos socialmente desviantes: uma análise do compromisso convencional e afiliação social. Tese de Doutorado, Departamento de Psicologia, Universidade Federal da Paraíba, João Pessoa.

Schlegel, R. P., D'Avernas, J. R., Zanna, M., Ditecco, D., \& Manske, S. R. (1987). Predicting alcohol use in young adult males: A comparison of the Fishbein-Ajzen model and Jessors' problem behavior theory. Drugs \& Society, 1(4), 7-19.

Scott, C. G. (1996). Understanding attitude change in developing effective substance abuse prevention programs for adolescents. School Counselor, 43(3), 187-195.

Secretaria Nacional Anti-Drogas. (2008). Danos causados pelo álcool aumentam gastos do SUS e despesas previdenciárias, diz MPF. Recuperado em 24 de dezembro de 2008, de http:// www.obid.senad.gov.br/portais/OBID/conteudo/web/noticia/ ler_noticia.php?id noticia $=102395$

Simons, J., \& Carey, K. B. (1998). A structural analysis of attitudes toward alcohol and marijuana use. Personality and Social Psychology Bulletin, 24(7), 727-35.

Simons, J., \& Carey, K. B. (2000). Attitudes toward marijuana use and drug-free experience: Relationships with behavior. Addictive Behaviors, 25(3), 323-31.

Simons, J. S., \& Gaher, R. M. (2004). Attitudes toward alcohol and drug-free experience among college students: Relationships with alcohol consumption and problems. American Journal of Drug and Alcohol Abuse, 31(2), 337-56.

Syre, T. R., Pesa, J. A., \& Cockley, D. (1999). Alcohol problems on college campuses escalate in 1997-1998: Time for action. College Student Journal, 33, 82-86.

Tabachnick, B. G., \& Fidell, L. S. (2001). Using multivariate statistics. Needham Heights, MA: Allyn \& Bacon.

Tavares, B. F., Béria, J. U., \& Lima, M. S. (2001). Prevalência do uso de drogas e desempenho escolar entre adolescentes. Revista de Saúde Pública, 35(2), 150-158.

Van De Vijer, F., \& Leung, K. (1997). Methods and data analysis for cross-cultural research. Thousand Oaks, CA: Sage.

Vargas, D. (2005). A construção de uma escala de atitudes frente ao álcool, ao alcoolismo e ao alcoolista: um estudo psicométrico. Tese de Doutorado, Escola de Enfermagem de Ribeirão Preto, Universidade de São Paulo, Ribeirão Preto, SP. 